\title{
So do you want to work overseas? Some guidance for medical educators working internationally
}

\author{
McKimm, J', McLean, M. ${ }^{2}$, Da Silva, A.S. ${ }^{1}$, Major, S. $^{3}$
}

\author{
${ }^{1}$ Swansea University School of Medicine, Swansea, Wales, UK, ${ }^{2}$ Faculty of Medicine \& Health \\ Sciences, Bond University, Gold Coast, Australia, ${ }^{3}$ Weill Cornell Medicine, Qatar
}

\begin{abstract}
Whilst many medical educators engage in international activities (such as conferences or projects), working internationally on a limited basis or more permanently will generally require adapting to different cultures and contexts. In some instances, this might be difficult. When considering working internationally, there are a number of personal and professional considerations. Personal considerations include motivation for wanting to work abroad, values and beliefs, the practicalities, as well as family involvement. Professional considerations include maintaining professional registration and adapting to different institutional work patterns and expectations, cultures and hierarchies. This article provides guidance for medical educators who are considering working in other countries and regions.
\end{abstract}

\section{Introduction}

As the world becomes smaller and more interconnected through cheaper travel, social media and migration, the medical education 'global village' is expanding in tandem with the globalization of the academic labour market (Kanter, 2010; McLean et al., 2013). 'Working' internationally often begins with international conferences or collaborating on international projects, which may or may not require travel to another country. These experiences provide safe opportunities to learn about other cultures and countries. A next step might be short-term contracts or consultancies, with the greatest cultural leap for individuals and their families being permanent emigration.

Our personal experiences and our research suggest that 'becoming' an international medical educator is a rich and reflexive process (McLean et al., 2013, 2014). This article provides guidance for those who may be contemplating working away from their 'home country' from the perspective of the individual educator. A related Opinion Piece, however, considers the implications for institutions which lose educators to other regions.

\section{Personal issues}

Think through why you want to work overseas

Major differences exist between a visit or shortterm project in another country and emigrating with family. Between these extremes, many opportunities may arise and, depending on your reasons for wanting to work overseas, you need to reflect on whether your skills set and career aspirations match what potential employers seek.

\section{Reflect on your 'cultural intelligence'}

Employment is only one part of a new culture. You will need to be tolerant of others' beliefs and practices, open to new experiences and also be willing to adapt aspects of your current life in order to 'fit in'. 


\section{Consider practical issues}

Balancing living and working in a new country whilst retaining the presence at home can be difficult, particularly with family commitments. Considerations include money, maintaining one or more homes, clinical practice, travel and visas. Depending on where you live, climate, living conditions, personal safety, food choices and internet access may impact on your lifestyle (Richardson \& Zikic, 2007).

\section{Ensure your family members are on board and check their needs}

If your family needs you at home, short-term contracts or projects may be the best. Your decision will depend on many factors including the age, number and schooling stage of your children, whether you have elderly dependents and your partner's occupation. Speak with families who have lived in the country for advice on how to settle your family. Some employers assist in finding work for professional partners, so explore this when applying for a new position.

\section{Find out as much as you can about the cultural context}

Moving to another country or region will invariably be in a new social and cultural context. Not being aware of subtleties can be embarrassing (even offensive) so talk to people who have lived and worked there. Even when working short-term or delivering video sessions or webinars, familiarize yourself with the cultural aspects of learning, the role of the teacher and students' expectations from you as these can vary considerably.

\section{Learn some of the language}

Even in countries where the language is the same as your own, you will need to learn cultural differences, nuances and vocabulary (e.g. British vs. Australian vs. American 'English'). Making an effort to learn a few key words in the language(s) of the country shows a willingness to integrate.

\section{Build and maintain support networks}

Personal and professional support networks help you to stay connected to the wider world and can lead to new opportunities for working internationally. Social media provides opportunities for keeping in touch with family, friends and colleagues. Locally, get involved with professional and community networks which will help you to adapt and assimilate into the new organization and culture.

\section{Professional issues}

\section{Plan how long you will stay}

It is important to decide how working internationally fits into your overall career plan, even if the plans change. Presenting at conferences and visiting specific institutions in certain countries may be a useful start because balancing your career with the needs of your partner or family may require compromises until circumstances change. Sometimes, because of your reputation or expertise, unexpected opportunities will arise, so staying flexible is advisable.

\section{Consider professional registration issues}

If you are clinically qualified, you will need to decide where and how you will maintain professional registration both in your home country and the new country. You will need to familiarize yourself with the health care system, health policies and ethical and medico-legal issues.

\section{Institutional issues}

\section{Find out as much as you can about the institution}

Request information's about the organization, its funding (public, private, endowment, charity), research opportunities, the curriculum, number of students and the staffing profile. This will help you to decide whether the move will allow you to continue on your career trajectory.

Have a clear job description and read
through your contract carefully before
signing

Before you sign a contract, make sure the terms and conditions are right (you may need to negotiate) and what the day-to-day expectations are from you. Clarify how your academic qualifications and experience compared with those in the host country and what development opportunities are available or may be expected from you.

\section{Visit the country and organization and ask for orientation}

Short-term work or a study visit to the institution which has offered you the position, can help with deciding on a move. If considering emigrating or accepting a long-term contract, a visit is essential to explore the locality, housing options, to talk to locals and potential colleagues and to see first-hand where you will 
work. Once you have arrived, a formal orientation Programme is essential. Our survey of 89 international medical educators identified that only a few employers offered orientation and this was generally inadequate (McLean et al., 2015).

\section{Conclusions}

Working internationally can be personally and professionally rewarding, enhancing your CV and providing opportunities that you may not have in your own country. It can, however, be difficult and lonely, so careful planning is required. We hope that this advice will be useful for those considering moving to a new context and will help to make your experience enjoyable.

\section{References}

Kanter S.L. (2010) International collaborations between medical schools: What are the benefits and risks? Academic Medicine, 85, 10, pp.1547-1548.

McLean, M., Da Silva, A., McKimm, J. \& Major, S. (2015) Medical educators working abroad: Who are they? MedEdPublish.

McLean, M., Major, S. \& McKimm, J. (2013) On being an international medical educator, In: Giardino, A.P., Giardino, E.R., [Eds.] Medical Education: Global Perspectives, Challenges and Future Directions. New York: Nova Science Publishers. pp. 17-26.

McLean, M., McKimm, J. \& Major, S. (2014) Medical educators working abroad: A pilot study of educators' experiences in the Middle East, Medical Teacher, 36,9, pp.757-764.

Richardson, J. \& Zikic, J. (2007) The darker side of an international academic career, Career Development International, 12,2, pp.164-186. 